[1] H. Adachi et al., Jpn. J. Appl. Phys. 42 (2003) L798.

[2] H. Adachi et al., Jpn. J. Appl. Phys. 43 (2004) L1376.

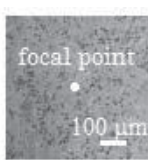

$-20 \mu \mathrm{s}$

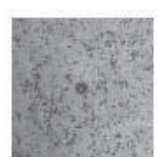

$0 \mu \mathrm{s}$

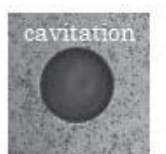

$13 \mu \mathrm{s}$

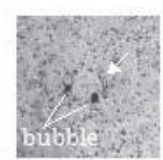

$73 \mu \mathrm{s}$
Fig. 1 The movement of microbeads by femtosecond laser irradiation

Keywords: protein crystallization, nucleation, laser technology
P04.01.55

Acta Cryst. (2008). A64, C247

\section{Wavelength dependence of the crystallization by the laser irradiation}

Hitoshi Hasenaka ${ }^{1}$, Ryota Murai ${ }^{1}$, Hiroshi Y. Yoshikawa ${ }^{1,2}$, Megumi Yamakami ${ }^{1,2}$, Tomoya Kitatani ${ }^{1,2}$, Shigeru Sugiyama ${ }^{1,2}$, Hiroaki Adachi $^{1,2,3}$, Kazufumi Takano ${ }^{1,2,3}$, Hiroyoshi Matsumura ${ }^{1,2,3}$, Satoshi Murakami ${ }^{2,3,4}$, Tsuyoshi Inoue ${ }^{1,2,3}$, Takatomo Sasaki ${ }^{1,3}$, Yusuke Mori ${ }^{1,2,3}$

${ }^{1}$ Osaka University, Electrical, Electronic and Information Engineering, 2-1 Yamadaoka, Suita, Osaka, 565-0871, Japan, ${ }^{2}$ CREST, JST,2-1 Yamadaoka, Suita, Osaka 565-0871, Japan, ${ }^{3}$ SOSHO Inc., Chuo-ku, Osaka, 541-0053, Japan, ${ }^{4}$ Institute of Scientific and Industrial Research, Osaka University, Ibaraki, Osaka, 567-0047, Japan, E-mail : hasenaka@cryst.eei.eng. osaka-u.ac.jp

Introduction : Light-induced crystallization have attracted attention as an application of temporal and spatial control of crystallization. We have proposed a new nucleation technique using a femtosecond laser at a wavelength of $780 \mathrm{~nm}$ and succeeded in producing highquality protein crystals [1]. In order to optimize a laser condition, we investigated wavelength dependence of the crystallization by the laser irradiation about nucleation probability. Experiment : We compared the probability of nucleation, when focused femtosecond laser beams were irradiated in protein solutions, such as Lysozyme and Glucose Isomerase, with various laser conditions (wavelength:260 nm, 390 $\mathrm{nm}, 780 \mathrm{~nm}$, energy: $13.5-94 \mu \mathrm{J} /$ pulse). Trials were carried out using a batch method at $23{ }^{\circ} \mathrm{C}$. At the same time we measured the strength of the impulse wave with shock wave sensor, and estimated deformations of solution. Result: In each wavelength, nucleation was promoted by femtosecond laser irradiation with certain energy level. However, nucleation probabilities were almost same in all wavelength of laser irradiated. These energy levels were comparable with threshold values of deformation of solution. Accordingly, nucleation was not dependent on wavelength of laser, but deformations of solution by the laser irradiation. From these results, we conclude that $780 \mathrm{~nm}$ laser is suitable for nucleation, because there is little denaturation of the protein by the laser irradiation at a fundamental wavelength of commercial femtosecond laser and there is no absorption to a plastic crystallization plate and a tape for sealing. [1] H. Adachi, et al., Jpn. J. Appl. Phys. 42 (2003) L798.

Keywords: wavelength, laser radiation, nucleation

\section{P04.01.56}

Acta Cryst. (2008). A64, C247-248

\section{Growth of large protein crystals for neutron crystallography by hanging a seed crystal}

Noriko Shimizu $^{1}$, Hiroshi Yoshikawa ${ }^{1,3}$, Motoyasu Adachi ${ }^{5}$, Taro Tamada ${ }^{5}$, Koushi Hidaka ${ }^{6}$, Yoshio Hayashi ${ }^{6}$, Yoshiaki Kiso ${ }^{6}$, Megumi Yamakami ${ }^{3}$, Tomoya Kitatani ${ }^{1,3}$, Shigeru Sugiyama ${ }^{1,3}$, Gen Sazaki ${ }^{1,3}$, Hiroaki Adachi ${ }^{1,3,4}$, Kazufumi Takano ${ }^{1,3,4}$,

Hiroyoshi Matsumura ${ }^{1,3,4}$, Satoshi Murakami ${ }^{2,3,4}$, Tsuyoshi Inoue ${ }^{1,3,4}$, Ryota Kuroki $^{5}$, Sasaki Takatomo ${ }^{1,4}$, Yusuke Mori ${ }^{1,3,4}$

${ }^{1}$ Osaka University, Electrical, Electronic and Information Engineering, 2-1 Yamadaoka, Suita, Osaka, 565-0871, Japan, ${ }^{2}$ Institute of Scientific and Industrial Research, Osaka University, Ibaraki, Osaka 567-0047, Japan, ${ }^{3}$ CREST-JST, Suita, Osaka 565-0871, Japan, ${ }^{4}$ SOSHO Inc., Chuo-ku, Osaka, 541-0053, Japan, ${ }^{5}$ Quantum Beam Science Directorate, Japan Atomic Energy Agency, Tokai, Ibaraki, 319-1195, Japan, ${ }^{6}$ Center for Frontier Research in Medicinal Science, Kyoto Pharmaceutical University, Kyoto, Kyoto 607-8412, Japan, E-mail : nshimizu@cryst.eei.eng.osaka-u. ac.jp 